\title{
Dependence of chaotic actuation dynamics of Casimir oscillators on optical properties and electrostatic effects
}

\author{
Fatemeh Tajik ${ }^{1,2}$, Mehdi Sedighi ${ }^{2}$, Amir Ali Masoudi ${ }^{1}$, Holger Waalkens $^{3}$, and George Palasantzas ${ }^{2, \text { a }}$ \\ ${ }^{1}$ Department of Physics, Alzahra University, Tehran 1993891167, Iran \\ 2 Zernike Institute for Advanced Materials, University of Groningen, Nijenborgh 4, 9747 AG Groningen, \\ The Netherlands \\ ${ }^{3}$ Johann Bernoulli Institute for Mathematics and Computer Science, University of Groningen, Nijenborgh 9, \\ 9747 AG Groningen, The Netherlands
}

Received 25 December 2017 / Received in final form 2 February 2018

Published online 30 April 2018

(C) The Author(s) 2018. This article is published with open access at Springerlink.com

\begin{abstract}
With Casimir and electrostatic forces playing a crucial role for the performance and stability of microelectromechanical systems (MEMS), the presence of chaotic behavior, which is often unavoidable, leads to device malfunction due to stiction. Therefore, we investigate here how the optical properties of different materials influence the chaotic behavior of electrostatic torsional MEMS due to changes in magnitude of the Casimir forces and torques. We consider the materials $\mathrm{Au}$, which is a good conductor, AIST, which is a phase change material being close to metal in the crystalline state, and finally doped SiC as a very poor conductor. For the conservative systems, there is no chaotic behavior and the analysis of phase portraits and bifurcation diagrams reveal the strong sensitivity of stable actuation dynamics on the material optical properties, while applied electrostatic potentials lead faster to instability and stiction for higher conductivity materials. For the driven systems, the Melnikov method is used to study the chaotic behavior. The results from this method are supported by the study of the contours of the transient time to stiction in the phase plane, which reveal a substantially increased chaotic behavior for higher conductivity materials, associated with stronger Casimir torques and applied electrostatic potentials.
\end{abstract}

\section{Introduction}

Dispersion forces, also known as van der Waals and Casimir forces, are omnipresent in nature and become dominant when the bodies are separated by distances smaller than $100 \mathrm{~nm}$ [1]. With the advancement in fabrication and miniaturization of MEMS technology towards nanoelectromechanical systems (NEMS) [2-9], deep understanding of stiction phenomena and correct estimation of the magnitude of the Casimir force is crucial for the analysis and design of MEMS/NEMS involving complex materials. This is because these systems have sufficiently large surface areas and gaps small enough for Casimir forces and torques to play a significant role causing device malfunction due to permanent adhesion, known as stiction, of moving components. Therefore, strategies to reduce stiction are widely studied in an attempt to ensure stable device performance, and long-term predictability for complex MEMS/NEMS designs.

One of the most prominent methods of device actuation is electrostatic, where inevitably Casimir forces and torques could play a role [3,10-14]. Although the electrostatic forces can be switched off when no potential is applied, the Casimir forces are omnipresent and

\footnotetext{
${ }^{a}$ e-mail: g.palasantzas@rug.nl
}

can influence the actuation dynamics of devices. The Casimir force was predicted by Casimir in 1948 [15] where he proved that two perfectly conducting parallel plates, separated by a gap $d$, attract each other via the force $F_{\text {Cas }}=\pi^{2} \hbar c / 240 d^{4}$ due to perturbation of vacuum fluctuations of the electromagnetic (EM) field. Here $\hbar$ and $c$ are the Planck constant and the speed of light, respectively. Soon after Lifshitz and co-workers $[16,17]$ considered the general case of dielectric plates by exploiting the fluctuation-dissipation theorem, which relates the dissipative properties of the plates (optical absorption by many microscopic dipoles) and the resulting EM fluctuations. In terms of the Lifshitz theory $[16,17]$ the van der Waals and Casimir forces are the short and long range limits, respectively, of the same force. In torsional systems these forces generate mechanical Casimir torques that have to be taken into account during the analysus of actuation dynamics.

Torsional MEMS, which find applications in torsional radio frequency $(\mathrm{RF})$ switches, tunable torsional capacitors, torsional micro mirrors, and high precision Casimir force measurements [2-6], are viewed as a cantilever type, where of the two electrodes one is fixed and the other is able to rotate around an axis [18]. By simply applying a voltage, both the electrostatic and the mechanical Casimir torques, which originate from the normal Casimir 


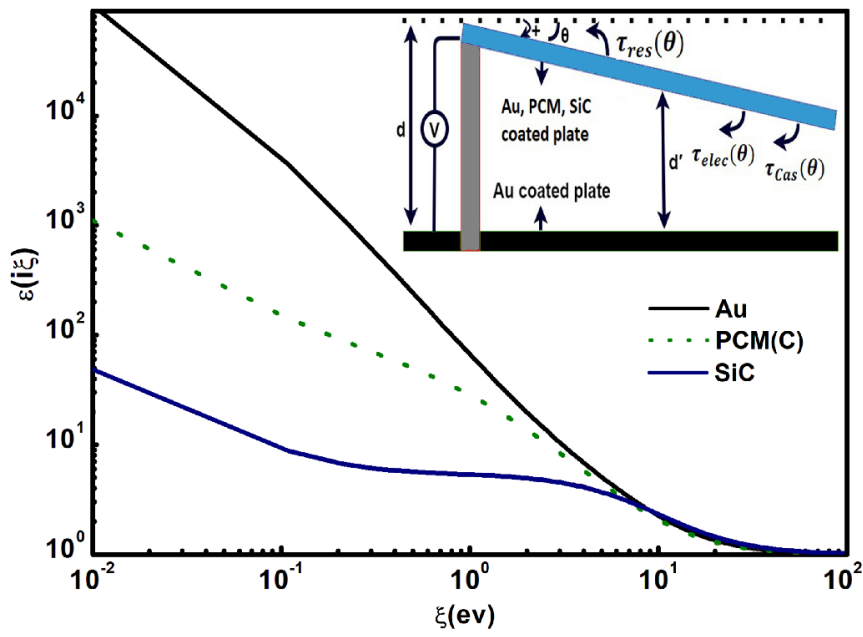

Fig. 1. Dielectric functions at imaginary frequencies $\varepsilon(i \xi)$ for $\mathrm{Au}, \mathrm{SiC}$, and crystalline (C) AIST (PCM (C)). The inset shows the schematic of the torsional system.

force [19-24], can rotate the movable electrode towards the fixed one. However, under certain conditions it can undergo jump-to-contact, which could lead to stiction [25]. Furthermore, the design of MEMS can be quite challenging due to the occurrence of chaotic behavior, which causes abrupt changes in their dynamical behavior, and as a result device malfunctions. Hence, more detailed knowledge about the phenomena of stiction and its relation to chaos is necessary to improve the performance and design of MEMS devices. So far, however, there is limited knowledge on how the Casimir forces-torques between actuating components at close proximity (typically less than $200 \mathrm{~nm}$ ) can lead to chaotic behavior when changing the strength of the force via the optical properties of interacting materials, and possibly in presence of applied electrostatic potentials.

Therefore, we investigate here theoretically how the change in optical properties of materials, corresponding to several orders of magnitude change in material conductivity and subsequently of the Casimir torque [26-29], can influence the chaotic behavior of torsional MEMS taking into account electrostatic torques. The choice of the materials in the present study (see Fig. 1) is motivated by requiring them to have the following properties: (i) the application of electrostatic voltage is feasible [5,6,29-36], (ii) they are used in actuating devices, and (iii) they show significantly diverse values of their conductivity ratio $\omega_{p}^{2} / \omega_{\tau}$ with $\omega_{p}$ the plasma frequency, and $\omega_{\tau}$ the damping factor in terms of the Drude model that is used to fit the low frequency optical data in Casimir studies [5,6,29-36]. The measured optical properties of these materials are used as input for the theory calculations of dynamic actuation.

\section{Modeling of dynamical system}

For our purpose, Au was used as a material due to its high conduction ratio $\omega_{p}^{2} /\left.\omega_{\tau}\right|_{\mathrm{Au}} \approx 1600 \mathrm{eV}$ and its frequent use in devices $[5,6,30]$. As an intermediate conductivity system we used the crystalline (C) state of the phase change material (PCM) AIST $\left(\mathrm{Ag}_{5} \mathrm{In}_{5} \mathrm{Sb}_{60} \mathrm{Te}_{30}\right)$. The latter is used in optical data storage (Blue-Rays, DVDs, etc.) and analysis of the optical data for Casimir studies, with a measured force contrast $\sim 25 \%$ between the amorphous (A) and crystalline (C) states, yields the conductivity ratio $\omega_{p}^{2} /\left.\omega_{\tau}\right|_{\operatorname{AIST}(\mathrm{C})}=10.1 \mathrm{eV}[29]$. As a poor conductor we use nitrogen doped $\mathrm{SiC}$, which is suitable for operation in harsh environments and an important element in Si-based technologies [34,35]. Analysis of optical data gives for $\mathrm{SiC}$ the conductivity ratio $\omega_{p}^{2} /\left.\omega_{\tau}\right|_{\mathrm{SiC}}=0.4 \mathrm{eV}$ [34]. The corresponding dielectric functions $\varepsilon(i \xi)$ at imaginary frequencies, which are necessary as input for the calculations of the Casimir force via Lifshitz theory (see Appendix A), are shown in Figure 1.

Furthermore, we consider the electrostatic torsional actuator shown in the inset of Figure 1, where only the upper plate is rotatable (and stiff enough to resist any buckling by the applied torques). The fixed plate is assumed to be coated by $\mathrm{Au}$, while the rotatable one by the materials of interest $\mathrm{Au}, \mathrm{SiC}$, and AIST (C) (with coating thickness in all cases more than $100 \mathrm{~nm}$ to ensure optically bulk materials) $[30,34]$. For simplicity, we consider flat plates because at short separations $(<100 \mathrm{~nm})$ nanoscale roughness can have significance influence [26,37-39]. The initial distance when the plates are parallel is assumed to be $d=200 \mathrm{~nm}$, and the system temperature $T=300 \mathrm{~K}$. The equation of motion for the torsional system is given by

$$
I_{0} \frac{d^{2} \theta}{d t^{2}}+\varepsilon I_{0} \frac{\omega_{0}}{Q} \frac{d \theta}{d t}=\tau_{\text {res }}+\tau_{\text {elec }}+\tau_{\text {Cas }}+\varepsilon \tau_{0} \cos (\omega t)
$$

where $I_{0}$ is the rotation moment of inertia of the rotating plate. The term $I_{0}(\omega / Q)(d \theta / d t)$ in equation (1) is due to the intrinsic energy dissipation of the moving plate with $Q$ the quality factor of the system. The frequency is assumed to be typical like in AFM cantilevers and MEMS [2-6]. The motion is conservative for $\varepsilon=0$, while for $\varepsilon=1$ we have forced non-conservative motion.

In equation (1) the torsional restoring torque $\tau_{\text {res }}$ is given by $\tau_{\text {res }}=k \theta$ with the torsional angle, which is considered positive as the plates move closer to each other. $k$ is the torsional spring constant around the support point allowing rotation of the rest of the beam [31-33,40-43]. $\tau_{\text {elec }}$ is the electrostatic torque when a voltage $V_{a}$ is applied between the two electrodes, and it is given by $[21,22]$

$$
\begin{aligned}
\tau_{\text {elec }}= & \frac{1}{2} \varepsilon_{0} L_{y}\left(V_{a}-V_{c}\right)^{2} \frac{1}{\sin ^{2}(\theta)}\left[\ln \left(\frac{d-L_{x} \sin (\theta)}{d}\right)\right. \\
& \left.+\frac{L_{x} \sin ^{2}(\theta)}{d-L_{x} \sin ^{2}(\theta)}\right]
\end{aligned}
$$

$V_{c}$ is the contact potential difference between the coating materials of the fixed and rotatable plate. In the following we will consider only the potential difference $V=V_{a}-V_{c}$ for the torque calculations. Finally the mechanical Casimir 

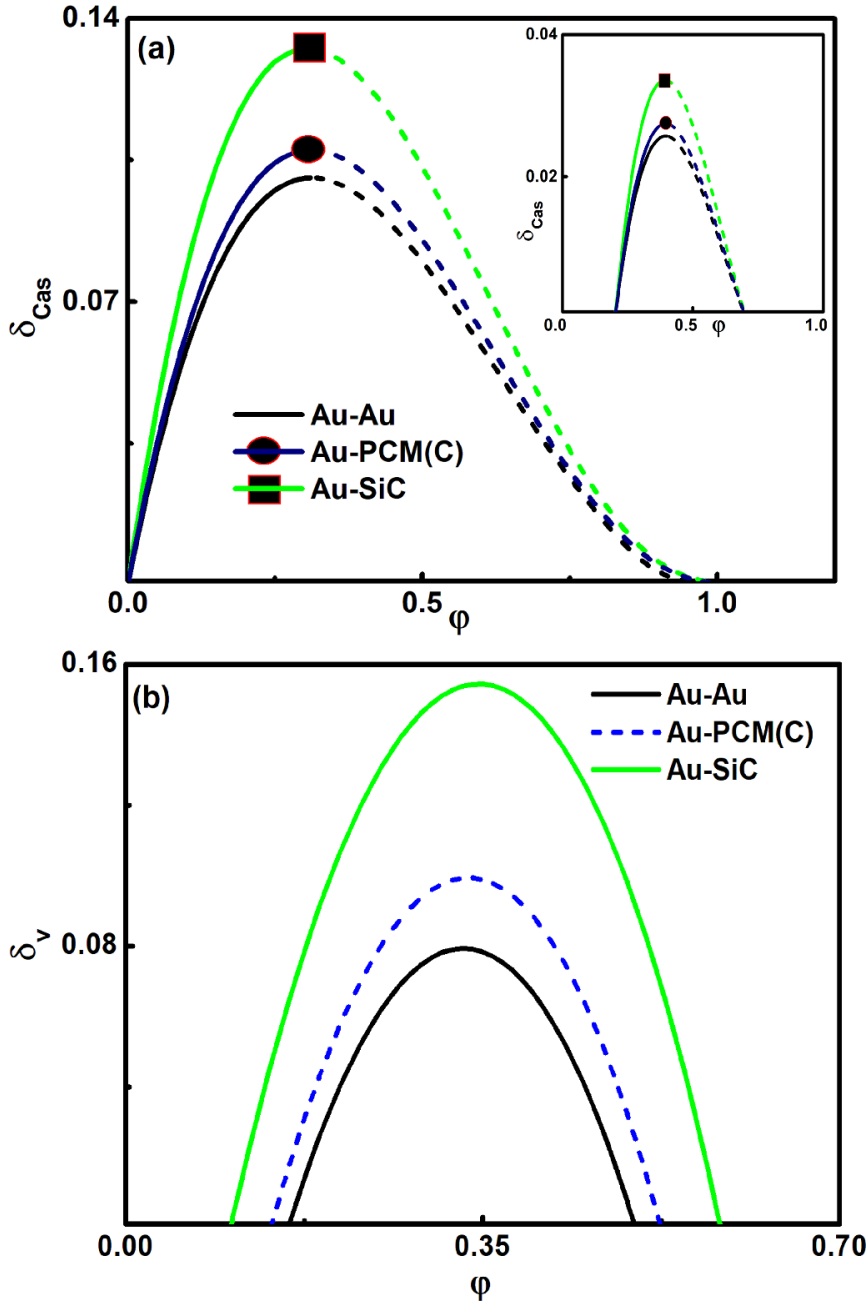

Fig. 2. (a) Bifurcation diagrams $\delta_{\text {Cas }}$ vs. with $\delta_{v}=0$ (the inset shows similar plots for $\delta_{v}=0.3$ ). The solid and dashed lines represent the stable and unstable points respectively. (b) Bifurcation diagrams $\delta_{v}$ vs. for $\delta_{\text {Cas }}=0.08$.

torque $\tau_{\text {Cas }}$, which acts on the rotating plate, is given by $[24]$

$$
\tau_{\mathrm{Cas}}=\int_{0}^{L_{x}} r F_{\mathrm{Cas}}\left(d^{\prime}\right) L_{y} d r
$$

where $F_{\text {Cas }}(d)$ is the Casimir force that is calculated using Lifshitz theory (see Appendix A), and $d^{\prime}=d-r \sin \theta$ with $d$ the distance for parallel plates of width and length $L_{x}$ and $L_{y}$. We choose $L_{x}=L_{y}=10 \mu \mathrm{m}$ and $d=200 \mathrm{~nm}$ so that the maximum torsional angle $\theta_{0}$ remains small $\left(\theta_{0}=d / L_{x}=0.02 \ll 1\right)$ in order to ignore also any buckling of the moving beam.

Furthermore, we introduce the Casimir bifurcation parameter $\delta_{\text {Cas }}=\tau_{\text {Cas }}^{m} / k \theta_{0}$, which represents the ratio of the minimum Casimir torque $\tau_{\text {Cas }}^{m}=\tau_{\text {Cas }}(\theta=0)$ and the maximum restoring torque $k \theta_{0}$ [44,45], and the bifurcation parameter for the electrostatic force $\delta_{v}=\left(\varepsilon_{0} V^{2} L_{y} L_{x}^{3}\right) /\left(2 k d^{3}\right)[13,14,20]$. Using $\delta_{\text {Cas }}$ and $\delta_{v}$ equation (1) assumes the more convenient form

$$
\begin{aligned}
\frac{d^{2} \varphi}{d T^{2}}+\varepsilon \frac{1}{Q} \frac{d \varphi}{d T}= & -\varphi+\delta_{v} \frac{1}{\varphi^{2}}\left[\ln (1-\varphi)+\frac{\varphi}{1-\varphi}\right] \\
& +\delta_{\text {Cas }}\left[\frac{\tau_{c a s}}{\tau_{\text {Cas }}^{m}}\right]+\varepsilon \frac{\tau_{0}}{\tau_{\text {res }}^{M a x}} \cos \left(\frac{\omega}{\omega_{0}} T\right)
\end{aligned}
$$

with $\varphi=\theta / \theta_{0}, T=\omega_{0} t$, and $I=I_{0} / k$.

\section{Results and discussion}

\subsection{Conservative system $(\varepsilon=0)$}

The equilibrium points for conservative motion are obtained by the condition $\tau_{\text {total }}=\tau_{\text {res }}+\tau_{\text {elec }}+\tau_{\text {Cas }}=0$. The latter yields from equation (4)

$$
-\varphi+\delta_{v} \frac{1}{\varphi^{2}}\left[\ln (1-\varphi)+\frac{\varphi}{1-\varphi}\right]+\delta_{\mathrm{Cas}}\left[\frac{\tau_{\mathrm{cas}}}{\tau_{\mathrm{Cas}}^{m}}\right]=0
$$

Figure 2 shows plots of $\delta_{\text {Cas }}, \delta_{v}$ vs. for all materials studied here. The solid lines in Figure 2a show stable regions where the restoring torque $\tau_{\text {res }}$ is strong enough to produce a stable equilibrium point near which the motion is periodic since $\delta_{\text {Cas }} \sim 1 / k$. The dash lines indicate unstable regions, where the equilibrium of the torsional system is unstable, and the moving beam undergoes stiction due to motion close to the fixed plate. The situation is qualitatively similar in presence of an electrostatic voltage (inset Fig. 2a). If the applied voltage increases then $\delta_{\text {Cas }}^{\mathrm{MAX}}$ also decreases. Due to the attractive nature of the electrostatic force, the device would require a higher restoring torque to preserve stable motion of the system. The presence of two equilibrium points occurs if $\delta_{\mathrm{Cas}}<\delta_{\mathrm{Cas}}^{\mathrm{MAX}}$. The equilibrium point closer to $\varphi=0$ (solid line) is stable and the other one closer to $\varphi=1$ (dashed line) is unstable. When $\delta_{\text {Cas }}$ reaches $\delta_{\mathrm{Cas}}^{\mathrm{MAX}}$ for the torsional system with higher conductivity materials, it is still $\delta_{\text {Cas }}<\delta_{\text {Cas }}^{\mathrm{MAX}}$ for the other less conductive materials yielding two equilibrium points and ensuring increased possibility for stable motion. Moreover, voltage bifurcation analysis gives useful information about the device dynamics. Figure $2 \mathrm{~b}$ shows the sensitive dependence of the bifurcation parameter $\delta_{v}$ on materials. Not only the maximum $\delta_{v}^{\mathrm{MAX}}$ decreases, but also the distance between the stable and unstable equilibrium points with increasing material conductivity. In all cases, the range of bifurcation parameters to produce stable periodic motion $\left(0<\delta_{\text {Cas }}<\delta_{\text {Cas }}^{\mathrm{MAX}}\right.$ and $\left.\delta_{v} \geq 0\right)$ decreases for increasing material conductivity.

Further information about the dynamics can be obtained from the phase diagrams $d \varphi / d t$ vs. $\varphi$. For a conservative system, the homoclinic orbit separates stable motion that manifests itself as continuous oscillation around the stable equilbirum from unstable motion across the unstable equilibrium point which leads to stiction. The homoclinic orbit is the orbit which connects the unstable equilibrium in the limit of infinite positive or negative time to itself. Hence, only the initial conditions in the region of the phase plane, which is enclosed by the homoclinic orbit, 

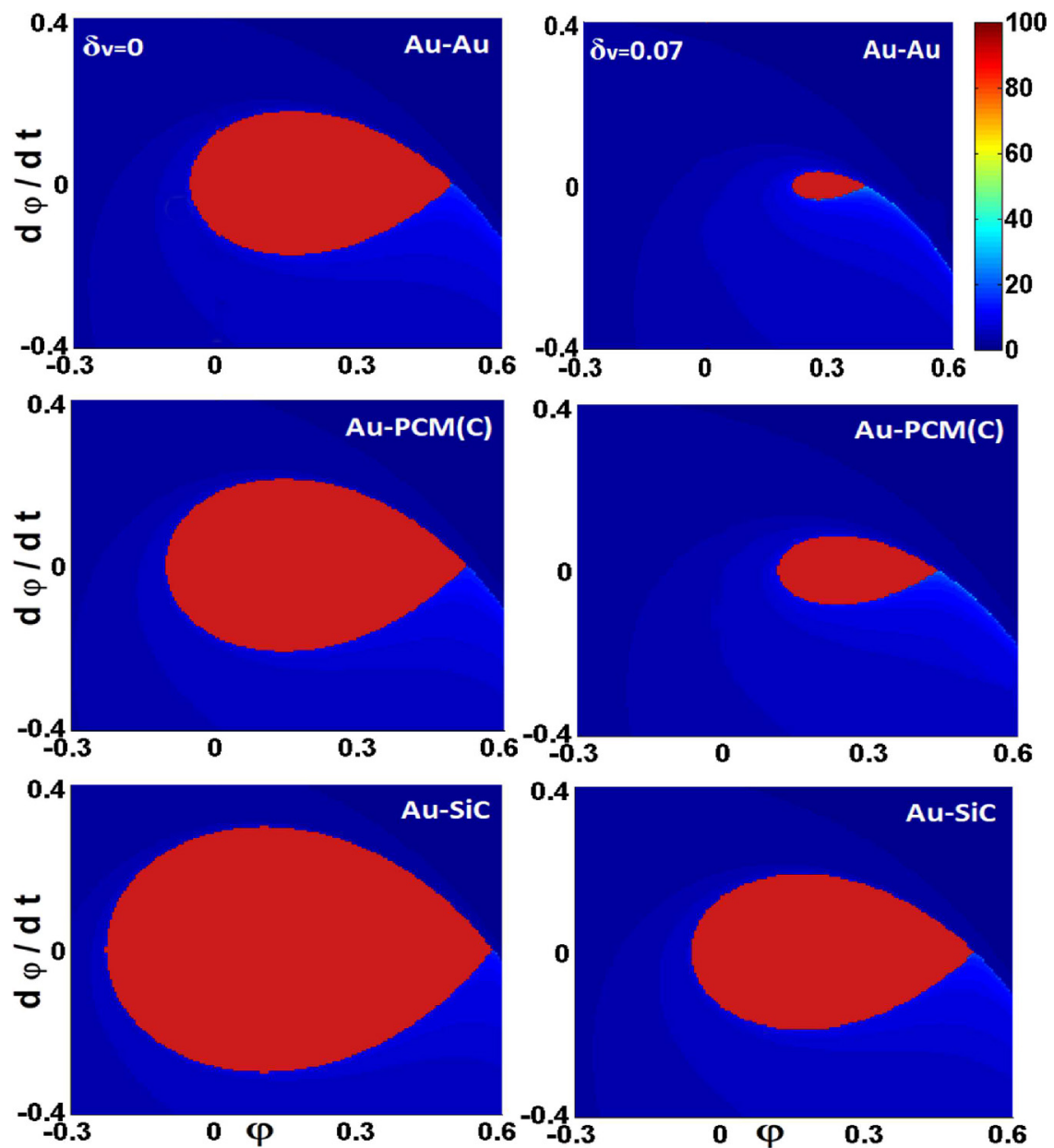

Fig. 3. Phase portrait $d \varphi / d t$ vs. $\varphi$ (with $\delta_{\text {Cas }}=0.08$, left column $\delta_{v}=0$, and right column $\delta_{v}=0.07$ ) for the conservative system. For the calculations we used $150 \times 150$ initial conditions $(\varphi, d \varphi / d t)$. The red region shows that initial condition for which the torsional device shows stable motion after 100 oscillations. The homoclinic orbit separates sharply stable and unstable solutions also reflecting the absence of chaotic behavior.

result in stable oscillatory motion. For any other initial conditions in the region outside the homoclinic orbit, the moving beam will perform unstable motion and collapse onto the ground plate within one period. As for any conservative system with one degree of freedom, the motion is not chaotic. However, according to Figure 3, for the less conductive material the size of the region enclosed by the homoclinic orbit is larger leading to a wider range for stable operation. In addition, any application of voltage strongly reduces the size of the region enclosed by the homoclinic orbit, and consequently the range of initial conditions that favor stable motion as the material conductivity increases.

\subsection{Non-conservative system $(\varepsilon=1)$}

Here we investigate the existence of chaotic behavior of the torsional system of finite quality factor $Q$ during forced oscillation via an applied external torque $\tau_{o} \cos (\omega t)$ [38]. In this case the the separatrix (homoclinic orbit) of the conservative system "splits". For the driven system, the unstable equilibrium turns into an unstable periodic orbit. The splitting of the separatrix means that the orbits which asymptotically reach the unstable periodic orbit in the forward and backward time directions no longer coincide. These orbits now form the unstable and stable manifolds of the unstable periodic orbit, respectively. Chaotic motion occurs if the stable and unstable manifolds have a transversal intersection. This condition can be investigated by the Melnikov method $[38,46]$. If we denote the homoclinic solution of the conservative system as $\varphi_{\mathrm{hom}}^{C}(T)$, then the Melnikov function for the torsional system is given by $[38,46]$

$$
\begin{aligned}
M\left(T_{0}\right)= & \frac{1}{Q} \int_{-\infty}^{+\infty}\left(\frac{d \varphi_{\mathrm{hom}}^{C}(T)}{d T}\right)^{2} d T+\frac{\tau_{0}}{\tau_{\text {res }}^{\mathrm{MAX}}} \\
& \times \int_{-\infty}^{+\infty} \frac{d \varphi_{\mathrm{hom}}^{C}(T)}{d T} \cos \left[\frac{\omega}{\omega_{0}}\left(T+T_{0}\right)\right] d T
\end{aligned}
$$

The stable and unstable manifolds have a transverse intersection if the Melnikov function has simple zeros, i.e. $M\left(T_{0}\right)=0$ and $M^{\prime}\left(T_{0}\right) \neq 0$. If $M\left(T_{0}\right)$ has no zeros, then the motion will not be chaotic. The conditions of nonsimple zeros, $M\left(T_{0}\right)=0$ and $M^{\prime}\left(T_{0}\right)=0$ gives the threshold 

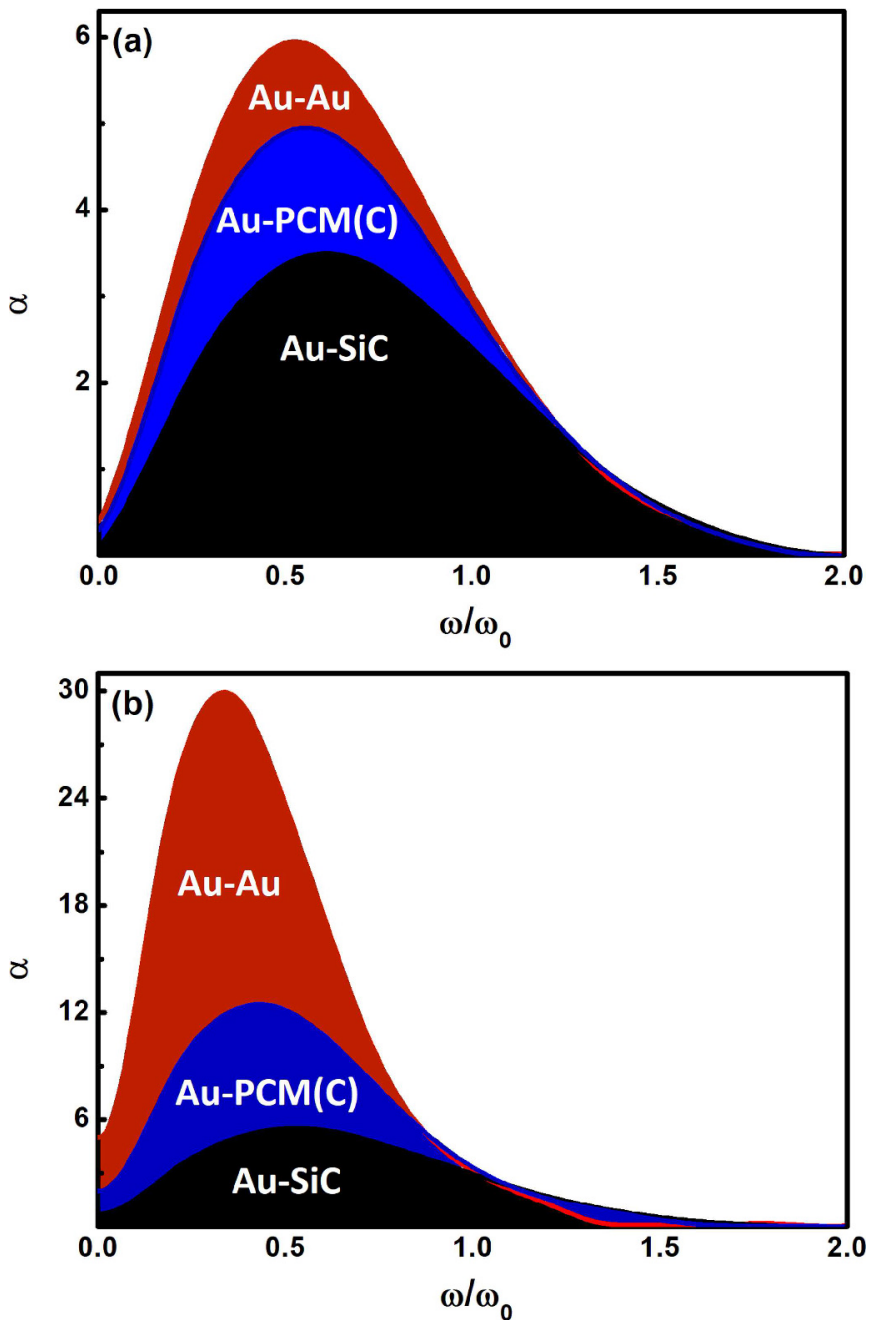

Fig. 4. Threshold curve $\alpha\left(=\gamma \omega_{0 \theta_{0}} / \tau_{0}\right)$ vs. driving frequency $\omega / \omega_{o}$ (with $\omega_{o}$ the natural frequency of the system). The area bellow the curve corresponds to parameters that lead to chaotic motion $\left(\delta_{\text {Cas }}=0.08\right.$, (a) $\delta_{v}=0$ and (b) $\left.\delta_{v}=0.07\right)$.

condition for chaotic motion $[38,46]$. If we define

$$
\begin{aligned}
& \mu_{\mathrm{hom}}^{c}=\int_{-\infty}^{+\infty}\left(\frac{d \varphi_{\mathrm{hom}}^{C}(T)}{d T}\right)^{2} d T \\
& \text { and } \beta(\omega)=\left|H\left[\mathfrak{R}\left(F\left\{\frac{d \varphi_{\mathrm{hom}}^{C}(T)}{d T}\right\}\right)\right]\right|,
\end{aligned}
$$

then the threshold condition for chaotic motion $\alpha=$ $\beta(\omega) / \mu_{\text {hom }}^{c}$ with $\alpha=\gamma \omega_{0 \theta_{0}} / \tau_{0}=(1 / Q)\left(\tau_{0} / \tau_{\text {res }}^{\mathrm{MAX}}\right)^{-1}$ obtains the form

$$
\begin{aligned}
\alpha= & \frac{\gamma \omega_{0 \theta_{0}}}{\tau_{0}}=\frac{\left|H\left[\Re\left(F\left\{\frac{d \varphi_{\text {hom }}^{C}(T)}{d T}\right\}\right)\right]\right|}{} \\
& \times \int_{-\infty}^{+\infty}\left(\frac{d \varphi_{\text {hom }}^{C}(T)}{d T}\right)^{2} d T,
\end{aligned}
$$

where $\gamma=I \omega_{o} / Q$, and $H[\ldots]$ denotes the Hilbert transform $[38,46]$.
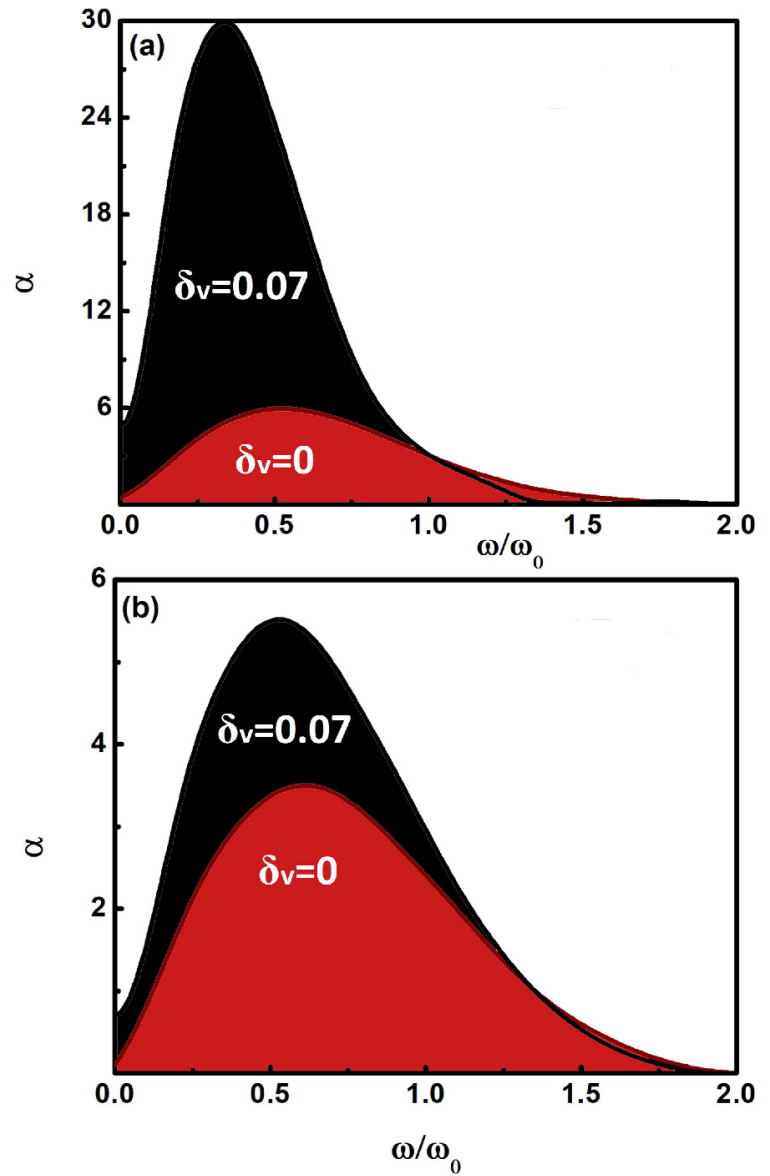

Fig. 5. Threshold curve $\alpha\left(\gamma \omega_{0 \theta_{0}} / \tau_{0}\right)$ vs. driving frequency $\omega / \omega_{o}$ (with $\omega_{o}$ the natural frequency of the system) for (a) $\mathrm{Au}-\mathrm{Au}$ and (b) Au-SiC. The area below the curve corresponds to parameters that lead to chaotic motion.

Figure 4 shows the threshold curves $\alpha=\gamma \omega_{0 \theta_{0}} / \tau_{0}$ vs. driving frequency ratio $\omega / \omega_{0}$. For large values of $\alpha$ (above the curve), the dissipation dominates the driving torque leading to regular motion that asymptotically approaches the stable periodic orbit resulting from the stable equilibrium point of the conservative system. However, for parameter values below the curve, the transversal intersections of the stable and unstable manifolds causes chaotic motion. Clearly for systems with higher conductivity, which lead to stronger Casimir torques, chaotic motion is more likely to occur as it is manifested by the larger area below the threshold curve. Figure $4 \mathrm{~b}$ shows the strong dependence of the region below the threshold curve, which corresponds to chaotic motion, on the applied voltage. The presence of an electrostatic torque changes the threshold curves, which is further amplified by the increasing material conductivity. According to Figure 5 the largest change for the threshold condition belongs to $\mathrm{Au}-\mathrm{Au}$ system, which has the highest conductivity, while for the $\mathrm{Au}$-Sic system it is drastically weaker for the same applied voltage.

Indeed, the chaotic behavior is shown by the contours of the transient times to stiction in the phase plane in Figure 6 for different values of the threshold parameter for 

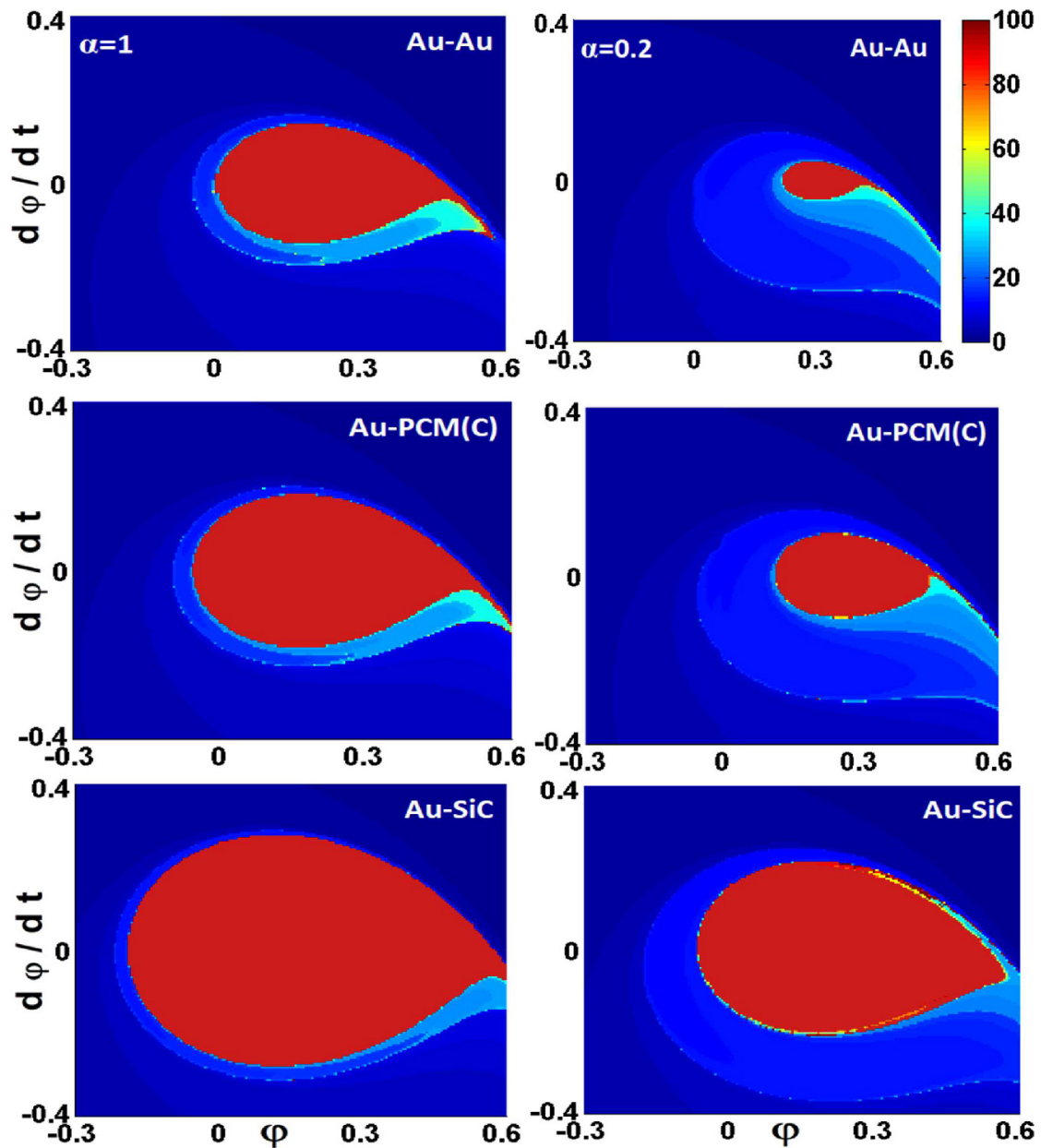

Fig. 6. Contour plot of the transient times to stiction in the phase plane $d \varphi / d t$ vs. $\left(\delta_{\text {Cas }}=0.08, \delta_{v}=0\right)$ for the non-conservative system for $\alpha=1$ (left column) and $\alpha=0.2$ (right column). For the calculations we used 150150 initial conditions $(\varphi, d \varphi / d t)$. The red region shows that initial condition for which the torsional device shows still stable motion after 100 oscillations. With decreasing the chaotic behavior increases, and the area of stable motion (red region) shrinks more for the systems with higher conductivity.

all materials studied here. If we compare with Figure 3, where chaotic motion does not occur, the latter plots show that chaotic motion takes place since there is no a simple smooth boundary between the red and the dark-blue regions. Chaotic behavior introduces significant risk for stiction, prohibiting long term prediction of the behavior of the oscillating system being more evident for material systems with higher conductivity or equivalently higher Casimir torques. In addition, as in Figure 5 for the threshold curves, the transient times to stiction in Figure 7 show the sensitive dependence of chaotic motion on the applied electrostatic potential for the $\mathrm{Au}-\mathrm{Au}$ and $\mathrm{Au}-\mathrm{SiC}$ systems. Again it is confirmed that any voltage application will strongly influence the chaotic behavior of the system having a dramatic effect for the higher conductivity materials (i.e. Au-Au system).

\section{Conclusions}

In conclusion, chaotic behavior, which is often unavoidable and leads to device malfunction, is strongly dependent on material conductivity and optical properties leading to different Casimir interactions, as well as on the applied electrostatic voltages. For conservative motion, phase portraits and bifurcation analysis show the strong sensitivity of actuation dynamics on the optical properties of interacting materials, where applied electrostatic forces lead faster to instability. For the driven systems, the Melnikov method in agreement with contours of the transient times to stiction revealed that an increasing material conductivity leads to stronger Casimir torques and subsequently more extensive chaotic behavior. The latter is strongly enhanced with applied electrostatic potentials. Since chaotic behavior leads to increased possibility for stiction prohibiting long term prediction of actuation dynamics, it becomes evident that characterization of the optical properties and conductivity of interacting materials is crucial for the design of dynamical microsystems.

GP and MS acknowledge support from the Zernike Institute of Advanced Materials, University of Groningen, The Netherlands. FT and AAM acknowledge support from the Department of Physics, Alzahra University, Iran. 

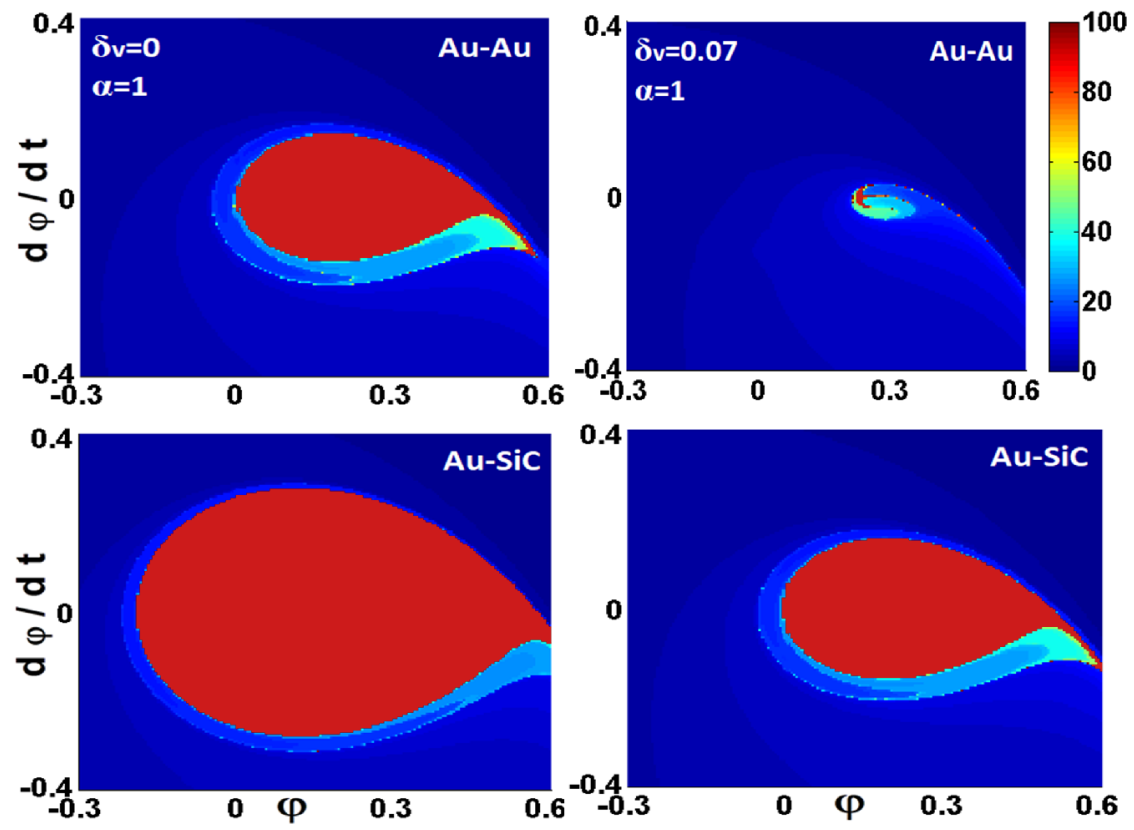

Fig. 7. Contour plot of the transient times to stiction in the phase plane $d \varphi / d t$ vs. $\varphi\left(\delta_{\text {Cas }}=0.08, \alpha=1\right)$ of the non-conservative system with $\delta_{v}=0$ (left column) and $\delta_{v}=0.07$ (right column) for $\mathrm{Au}-\mathrm{Au}$ and $\mathrm{Au}-\mathrm{SiC}$ systems. For the calculations we used $150 \times 150$ initial conditions $(\varphi, d \varphi / d t)$. The red region shows the initial conditions for which the torsional device shows stable motion after 100 oscillations. With oscillating frequency $\omega / \omega_{0}=0.5$. With increasing $\delta_{v}$ (or equivalently applied voltage) the chaotic behavior increases, and the area of stable motion shrinks more for the systems with higher conductivity and applied potential.

\section{Author contribution statement}

All authors contributed equally to the paper.

Open Access This is an open access article distributed under the terms of the Creative Commons Attribution License (http://creativecommons.org/licenses/by/4.0), which permits unrestricted use, distribution, and reproduction in any medium, provided the original work is properly cited.

\section{Appendix A: Brief Lifshitz theory and dielectric function of materials with extrapolations}

The Casimir force $F_{\text {Cas }}(d)$ in equation (3) is given by $[16,17]$

$$
\begin{aligned}
F_{\text {Cas }}(d)= & \frac{k_{B} T}{\pi} \sum^{\prime}{ }_{l=0} \sum_{\nu}=T E, T M \int_{0}^{\infty} d k_{\perp} k_{\perp} k_{0} \\
& \times \frac{r_{\nu}^{(1)} r_{\nu}^{(2)} \exp \left(-2 k_{0} d\right)}{1-r_{\nu}^{(1)} r_{\nu}^{(2)} \exp \left(-2 k_{0} d\right)}
\end{aligned}
$$

The prime in the first summation indicates that the term corresponding to $l=0$ should be multiplied with a factor $1 / 2$. The Fresnel reflection coefficients are given by $r_{\mathrm{TE}}^{(i)}=\left(k_{0}-k_{i}\right) /\left(k_{0}+k_{i}\right)$ and $r_{\mathrm{TM}}^{(i)}=$ $\left(\varepsilon_{i} k_{0}-\varepsilon_{0} k_{i}\right) /\left(\varepsilon_{i} k_{0}+\varepsilon_{0} k_{i}\right)$ for the transvers electric
(TE) and magnetic (TM) field polarizations, respectively. $k_{i}(i=0,1,2)=\sqrt{\varepsilon_{i}\left(i \xi_{l}\right)+k_{\perp}^{2}}$ represents the outoff plane wave vector in the gap between the interacting plates $\left(k_{0}\right)$ and in each of the interacting plates $\left(k_{i=(1,2)}\right)$. $k_{\perp}$ is the in-plane wave vector.

The function $\varepsilon(i \xi)$ is the dielectric function evaluated at imaginary frequencies, which is the necessary input for calculating the Casimir force between real materials using Lifshitz theory. The latter is given by $[16,17]$

$$
\varepsilon(i \xi)=1+\frac{2}{\pi} \int_{0}^{\infty} \frac{\omega \varepsilon^{\prime \prime}(\omega)}{\omega^{2}+\xi^{2}} d \omega .
$$

For the calculation of the integral in equation (A.2) one needs the measured data for the imaginary part $\varepsilon^{\prime \prime}(\omega)$ of the frequency dependent dielectric function $\varepsilon(\omega)$. The materials were optically characterized by ellipsometry over a wide range of frequencies at J. A.Woollam Co. using the VUV-VASE $(0.5-9.34 \mathrm{eV})$ and IR-VASE $(0.03-0.5 \mathrm{eV}))$ $[26,29,34,35]$. In any case the experimental data for the imaginary part $\varepsilon^{\prime \prime}(\omega)$ of the dielectric function cover only a limiting range of frequencies $\omega_{1}(=0.03 \mathrm{eV})<\omega<$ $\omega_{2}(=8.9 \mathrm{eV})$. Therefore, for the low optical frequencies $\left(\omega<\omega_{1}\right)$ we extrapolated using the imaginary part of the Drude model $[26,29,34,35]$

$$
\varepsilon^{\prime \prime}{ }_{L}(\omega)=\frac{\omega_{p}^{2} \omega_{\tau}}{\omega}\left(\omega^{2}+\omega_{\tau}^{2}\right),
$$

where $\omega_{p}$ is the plasma frequency, and $\omega_{\tau}$ is the relaxation frequency. Furthermore, for the high optical frequencies 
$\left(\omega>\omega_{2}\right)$ we extrapolated using $[26,29,34,35]$

$$
\varepsilon^{\prime \prime}{ }_{H}(\omega)=\frac{A}{\omega^{3}} .
$$

Finally, using equations (A.2)-(A.4), $\varepsilon(i \xi)$ is given by

$$
\begin{aligned}
\varepsilon(i \xi)_{C}= & 1+\frac{2}{\pi} \int_{\omega_{1}}^{\omega_{2}} \frac{\omega \varepsilon^{\prime \prime}{ }_{\exp }(\omega)}{\omega^{2}+\xi^{2}} d \omega \\
& +\Delta_{L} \varepsilon(i \xi)+\Delta_{H} \varepsilon(i \xi),
\end{aligned}
$$

with

$$
\begin{aligned}
& \Delta_{L} \varepsilon(i \xi)=\frac{2}{\pi} \int_{0}^{\omega_{1}} \frac{\omega \varepsilon^{\prime \prime}{ }_{L}(\omega)}{\omega^{2}+\xi^{2}} d \omega, \\
& \text { and } \Delta_{H} \varepsilon(i \xi)=\frac{2}{\pi} \int_{\omega_{2}}^{\infty} \frac{\omega \varepsilon^{\prime \prime}{ }_{H}(\omega)}{\omega^{2}+\xi^{2}} d \omega .
\end{aligned}
$$

\section{References}

1. J. Israelachvili, Intermolecular and surface forces (Academic, New York, 1992)

2. A.W. Rodriguez, F. Capasso, S.G. Johnson, Nat. Photonics 5, 211 (2011)

3. F. Capasso, J.N. Munday, D. Iannuzzi, H.B. Chan, IEEE J. Sel. Top. Quant. Electron. 13, 400 (2007)

4. M. Bordag, G.L. Klimchitskaya, U. Mohideen, V.M. Mostepanenko, Advances in the Casimir effect (Oxford University Press, New York, 2009)

5. S.R. Decca, D. López, E. Fischbach, G.L. Klimchitskaya, D.E. Krause, V.M. Mostepanenko, Ann. Phys. 318, 37 (2005)

6. S.R. Decca, D. López, E. Fischbach, G.L. Klimchitskaya, D.E. Krause, V.M. Mostepanenko, Phys. Rev. D 75, 077101 (2007)

7. A. Ashourvan, M.F. Miri, R. Golestanian, Phys. Rev. Lett. 98, $140801(2014)$

8. M.F. Miri, R. Golestanian, Appl. Phys. Lett. 92, 113103 (2011)

9. A. Ashourvan, M.F. Miri, R. Golestanian, Phys. Rev. E. 75, 040103 (2007)

10. P. Ball, Nature 447, 77 (2007)

11. G. Palasantzas, J.Th.M. DeHosson, Phys. Rev. B. 72, 121409 (2005)

12. G. Palasantzas, J.Th.M. DeHosson, Phys. Rev. B. 72, 115426 (2005)

13. F. Tajik, M. Sedighi, M. Khorrami, A.A. Masoudi, G. Palasantzas, Phys. Rev. E 96, 042215 (2017)

14. F. Tajik, M. Sedighi, G. Palasantzas, J. Appl. Phys. 121, $174302(2017)$

15. H.B.G. Casimir, Proc. K. Ned. Akad. Wet. 51, 793 (1948)

16. E.M. Lifshitz, Sov. Phys. JETP 2, 73 (1956)

17. I.E. Dzyaloshinskii, E.M. Lifshitz, L.P. Pitaevskii, Sov. Phys. Usp. 4, 153 (1961)

18. O. Bochobza-Degani, Y. Nemirovsky, Sens. Actuators A 97-98, 569 (2002)
19. Y. Nemirovsky, O. Degani, J. Microelectromech. Syst. 10, $601(2001)$

20. O. Degani, Y. Nemirovsky, J. Microelectromech. Syst. 11, $20(2002)$

21. J.G. Guo, Y.P. Zhao, Int. J. Solids Struct. 43, 675 (2006)

22. W.H. Lin, Y.P. Zhao, J. Phys. D: Appl. Phys. 40, 1649 (2007)

23. R. Satter, F. Plötz, G. Fattinger, G. Wachutka, Sens. Actuators A 97-98, 337 (2002)

24. W.H. Lin, Y.P. Zhao, Chaos Solitons Fractals 23, 1777 (2005)

25. R. Maboudian, R.T. Howe, J. Vac. Sci. Technol. B 15, 1 (1997)

26. G. Palasantzas, V.B. Svetovoy, P.J. van Zwol, Int. J. Mod. Phys. B 24, 6013 (2010)

27. F. Chen, G.L. Klimchitskaya, V.M. Mostepanenko, U.M. Mohideen, Opt. Express 15, 4823 (2007)

28. C.C. Chang, A.A. Banishev, G.L. Klimchitskaya, V.M. Mostepanenko, U. Mohideen, Phys. Rev. Lett. 107, 090403 (2011)

29. G. Torricelli, P.J. van Zwol, O. Shpak, G. Palasantzas, V.B. Svetovoy, C. Binns, B.J. Kooi, P. Jost, M. Wuttig, Adv. Funct. Mater. 22, 3729 (2012)

30. V.B. Svetovoy, P.J. van Zwol, G. Palasantzas, J.Th.M. DeHosson, Phys. Rev. B. 77, 035439 (2008)

31. S.K. Lamoreaux, Phys. Rev. Lett. 78, 5 (1997)

32. S.K. Lamoreaux, Rep. Progr. Phys. 68, 201 (2005)

33. H.B. Chan, V.A. Aksyuk, R.N. Kleiman, D.J. Bishop, F. Capasso, Phys. Rev. Lett. 87, 211801 (2001)

34. M. Sedighi, V.B. Svetovoy, W.H. Broer, G. Palasantzas, Phys. Rev. B 89, 195440 (2014)

35. M. Sedighi, V.B. Svetovoy, G. Palasantzas, Phys. Rev. B 93, 085434 (2016)

36. M. Wutting, N.Y. Yamada, Nat. Mater. 6, 824 (2007)

37. W. Broer, G. Palasantzas, J. Knoester, V.B. Svetovoy, Phys. Rev. B 87, 125413 (2013)

38. W. Broer, H. Waalkens, V.B. Svetovoy, J. Knoester, G. Palasantzas, Phys. Rev. Appl. 4, 054016 (2015)

39. V.B. Svetovoy, G. Palasantzas, Adv. Colloid Interface Sci. 216, 1 (2015)

40. F. Intravaia, A. Koev, I.W. Jun, A.A. Talin, P.S. Davids, R.S. Decca, V.A. Aksyuk, D.A.R. Dalvit, D. Lopez, Nat. Commun. 4, 2515 (2013)

41. R.S. Decca, D. Lopez, E. Fischbach, G.L. Klimchitskaya, D.E. Krause, V.M. Mostepanenko, Ann. Phys. 318, 37 (2005)

42. S.R. Decca, D. López, E. Fischbach, G.L. Klimchitskaya, D.E. Krause, V.M. Mostepanenko, Phys. Rev. D 75, 077101 (2007)

43. O. Degani, E. Socher, A. Lipson, T. Lejtner, D.J. Setter, Sh. Kaldor, Y. Nemirovsky, J. Microelectromech. Syst. 7, 373 (1998)

44. M. Sedighi, W.H. Broer, G. Palasantzas, B.J. Kooi, Phys. Rev. B 88, 165423 (2013)

45. S. Cui, Y.C. Soh, J. Microelectromech. Syst. 19, 1153 (2010)

46. J. Guckenheimer, P. Holmes, Nonlinear oscillations, dynamical systems, and bifurcations of vector fields (Springer, Berlin, Heidelberg, New York, 1983) 